\title{
THE SET OF NONPRINCIPAL ORBITS OF AN ACTION ON $E^{n}$
}

\author{
GLEN E. BREDON
}

The purpose of this note is to prove the following fact which was conjectured by Frank Raymond on p. 352 of [4].

Theorem. Let $G$ be a compact Lie group (not necessarily connected) acting on euclidean space $E^{n}$. Suppose that there is at least one stationary point. Then the set of points on nonprincipal orbits is connected.

(As Raymond notes, this is not hard to prove for abelian groups $G$. Also, it is known to be false without the hypothesis of the existence of a stationary point, even for $G=Z_{6}$; see [3].)

Actually, this theorem is a fairly easy consequence of the following result, which was essentially proved by us in [1, Chapter XV, Lemma 2.7] and [2, Lemma 2.2]. It is a generalization of the wellknown fact that the orbits of the action of the Weyl group on the Lie algebra of a maximal torus of a compact Lie group $G$ correspond in a one-one manner to the orbits of the adjoint representation of $G$ with the regular points also corresponding.

Theorem. Let $M^{n}$ be either $E^{n}$ or $S^{n}$ and let $G$ be a compact Lie group acting on $M^{n}$. Let $(H)$ be the principal orbit type and let $T$ be a maximal torus of $H$. Then the natural map

$$
f: \frac{F\left(T, M^{n}\right)}{N(T)} \rightarrow \frac{M^{n}}{G}
$$

is a homeomorphism and takes the set of principal orbits of the $N(T) / T$ action on $F\left(T, M^{n}\right)$ onto those of the action of $G$ on $M^{n}$.

Here $N(T)$ is the normalizer of $T$ in $G$ and $F\left(T, M^{n}\right)$ denotes the set of stationary points for $T$ on $M^{n}$. Note that the action of $N(T) / T$ on $F\left(T, M^{n}\right)$ may not be effective, but this will not concern us.

Actually the references cited prove this result in a local sense about a fixed point and with $M^{n}$ any generalized manifold over the integers. However, applying this local result to a neighborhood of the vertex of the open cone over $S^{n}$ gives the global result for $S^{n}$, and passage to the one point compactification gives the global result for $E^{n}$.

To prove Raymond's conjecture, we may take 0 to be a stationary point. Let $C$ be the set of points on nonprincipal orbits and let $x \in C$;

Received by the editors March 7, 1969. 
that is, $H$ is conjugate to a proper subgroup of the isotropy group $G_{x}$. Without loss of generality we may take $H$ to be a proper subgroup of $G_{x}$. Then $x \in F\left(T, E^{n}\right)$ and the $N(T) / T$-orbit of $x$ on $F\left(T, E^{n}\right)$ is not principal for this action. Let $G^{\prime}=N(T) / T$ and let $\left(H^{\prime}\right)$ be the principal orbit type of $G^{\prime}$ on $F\left(T, E^{n}\right)$. Then we may take $H^{\prime}$ to be a proper subgroup of $G_{x}^{\prime}$. Note that $H^{\prime}$ is finite. Now if $G_{x}^{\prime}$ is not finite then it contains a circle subgroup $S$ and then $F\left(S, F\left(T, E^{n}\right)\right)$ is a connected (in fact, acyclic) set containing 0 and $x$ and contained in $C$. Similarly, suppose that $G_{x}^{\prime}$ is finite. Then there is a prime power $p^{k}$ dividing ord $\left(G_{x}^{\prime}\right)$ but not dividing ord $\left(H^{\prime}\right)$. Let $P$ be a $p$-Sylow subgroup of $G_{x}^{\prime}$. Then, since $P$ is not conjugate to a subgroup of $H^{\prime}$, we see that $F\left(P, F\left(T, E^{n}\right)\right)$ is contained in $C$ and, by the Smith theorems, this is a connected set containing 0 and $x$.

\section{REFERENCES}

1. A. Borel et. al., Seminar on transformation groups, Ann. of Math. Studies No. 46, Princeton Univ. Press, Princeton, N. J., 1960.

2. G. E. Bredon, On the structure of orbit spaces of generalized manifolds, Trans. Amer. Math. Soc. 100 (1961), 162-196.

3. J. M. Kister, Differentiable periodic actions on $E^{8}$ without fixed points, Amer. J. Math. 85 (1963), 316-319.

4. P. S. Mostert, (editor), Proc. Conference on Transformation Groups, SpringerVerlag, New York, 1968.

RUTGERS UNIVERSITY 